\title{
On the Exponential Inequality for Weighted Sums of a Class of Linearly Negative Quadrant Dependent Random Variables
}

\author{
Guodong Xing and Shanchao Yang \\ School of Mathematics and Statistics, Guangxi Normal University, Guilin 541004, China \\ Correspondence should be addressed to Shanchao Yang; shanchaoyang11@sina.com
}

Received 24 August 2013; Accepted 20 November 2013; Published 27 January 2014

Academic Editors: K. Barbé, N. Ganikhodjaev, and A. Kabán

Copyright (C) 2014 G. Xing and S. Yang. This is an open access article distributed under the Creative Commons Attribution License, which permits unrestricted use, distribution, and reproduction in any medium, provided the original work is properly cited.

The exponential inequality for weighted sums of a class of linearly negative quadrant dependent random variables is established, which extends and improves the corresponding ones obtained by Ko et al. (2007) and Jabbari et al. (2009). In addition, we also give the relevant precise asymptotics.

\section{Introduction}

Lehmann [1] introduced a natural definition of negative dependence: two random variables $X$ and $Y$ are said to be negative quadrant dependent (NQD, say) if $P(X>x, Y>$ $y) \leq P(X>x) P(Y>y)$ for all $x, y \in \mathbf{R}$. Based on the concept of NQD, another notion of negative dependence was formulated by Newman [2] as follows: a sequence $\left\{X_{i}, 1 \leq\right.$ $i \leq n\}$ of random variables is said to be linearly negative quadrant dependent (LNQD, say) if, for any disjoint subsets $A_{1}$ and $A_{2}$ of $\{1,2, \ldots, n\}$ and positive $r_{j}$ 's, $\sum_{i \in A_{1}} r_{i} X_{i}$, and $\sum_{j \in A_{2}} r_{j} X_{j}$ are NQD. Recall that a finite family of random variables $\left\{X_{i}, 1 \leq i \leq n\right\}$ is said to be negatively associated (NA, say) if, for every pair of disjoint subsets $B_{1}$ and $B_{2}$ of $\{1,2, \ldots, n\}$,

$$
\operatorname{Cov}\left\{f_{1}\left(X_{i}, i \in B_{1}\right), f_{2}\left(X_{j}, j \in B_{2}\right)\right\} \leq 0,
$$

whenever $f_{1}$ and $f_{2}$ are coordinatewise increasing and the covariance exists. An infinite family is NA if every finite subfamily is NA. The concept of negative association was introduced by Joag-Dev and Proschan [3]. It is obvious to observe that NA sequences are LNQD and LNQD sequences are not necessarily NA, as it can be seen from the examples in Newman [2] or Joag-Dev and Proschan [3]. Hence, it is of interest to investigate the exponential inequality and its relevant result for LNQD sequences.

It is well-known that the exponential inequalities for partial sum $\sum_{i=1}^{n}\left(X_{i}-E X_{i}\right)$ play a very important role in various proofs of limit theorems. One can refer to Yang and Wang [4], T.-S. Kim and H.-C. Kim [5], Sung [6], Jabbari et al. [7], Xing et al. [8], Sung [9], and so on for further comprehension. As for the limit results about LNQD sequence, one can refer to Newman [2], Zhang [10], H. Kim and T. Kim [11], Wang and Zhang [12], and references therein.

Recently, Ko et al. [13] gave a Bernstein-Hoeffding type inequality for uniformly bounded LNQD random variables, by which they obtained the almost sure convergence rate $O(1) n^{-1 / 2}\left(p_{n} \log n\right)^{1 / 2}$ of $\sum_{i=1}^{n} X_{i} / n$, where $p_{n} \rightarrow \infty$ as $n \rightarrow$ $\infty$. Motivated by the paper above, we establish the exponential inequality for weighted sums of uniformly bounded LNQD random variables. The result obtained extends and improves the corresponding ones given by Ko et al. [13] and Jabbari et al. [7]. Furthermore, we give the precise asymptotics with respect to the rate $n^{-1 / 2}(\log n)^{1 / 2}$.

Throughout this paper, we always suppose that $C$ and $C_{1}$ denote positive constants independent of $n$ but whose value may vary over cases, $[x]$ denotes the integral part of $x, S_{n}=$ $\sum_{i=1}^{n} X_{i}, \sigma_{n}^{2}=E S_{n}^{2}$, and $u(n)=\sup _{i \geq 1} \sum_{j:|j-i| \geq n}\left|\operatorname{Cov}\left(X_{i}, X_{j}\right)\right|$ and denote $\log n=\ln (n \vee e)$. This paper is organized 
as follows. Section 2 contains our main results. Section 3 contains the corresponding proofs.

\section{Main Results}

In this section, our main results will be given. For formulation of the theorems obtained, some assumptions are needed, which are listed below.

(A1) Let $\left\{X_{i}, i \geq 1\right\}$ be a sequence of stationary LNQD random variables with $\left|X_{i}\right| \leq M$ and let $\left\{a_{n i}: 1 \leq i \leq\right.$ $n, n \geq 1\}$ be a triangular array of numbers satisfying $\left|a_{n i}\right| \leq M_{1}$, where $M$ and $M_{1}$ are generic positive constants.

(A2) Let $\left\{p_{n}, n \geq 1\right\}$ be a positive integer sequence satisfying $p_{n} \leq n / 2$ and $p_{n} \rightarrow \infty$ as $n \rightarrow \infty$.

Theorem 1. Suppose that the assumption (A1) holds. Then for any $0<\varepsilon<1$, one has

$$
P\left(\left|\sum_{i=1}^{n} a_{n i}\left(X_{i}-E X_{i}\right)\right|>n \varepsilon\right) \leq C_{1} \exp \left\{-\frac{n \varepsilon^{2}}{16 C}\right\},
$$

where $C_{1}$ and $C$ are positive constants.

Taking

$$
\varepsilon=\varepsilon_{n}:=4 \sqrt{\frac{(\alpha C \log n)}{n}}, \quad \text { for some } \alpha>1 \text {, }
$$

in Theorem 1, then by Borel-Cantelli lemma, we have

Corollary 2. Assume that the assumptions (A1) and (A2) hold. Let $p_{n} \leq \sqrt{C n /\left(\alpha M^{2} \log n\right)}$, for some $\alpha>1$. Suppose that $\varepsilon$ is as in (3). Then one has

$$
\sum_{i=1}^{n} a_{n i}\left(X_{i}-E X_{i}\right)=O(1)(n \log n)^{1 / 2} \quad \text { a.s. }
$$

In particular, one has

$$
\sum_{i=1}^{n}\left(X_{i}-E X_{i}\right)=O(1)(n \log n)^{1 / 2} \quad \text { a.s. }
$$

when $a_{n i}=1$ for $1 \leq i \leq n$.

Remark 3. (1) Theorem 1 generalizes Theorem 2.1 in Ko et al. [13] to weighted case. On the other hand, by (5), we can obtain that the strong convergence rate of $\sum_{i=1}^{n}\left(X_{i}-\right.$ $\left.E X_{i}\right) / n$ is $O(1) n^{-1 / 2} \log ^{1 / 2} n$, which is obviously faster than the corresponding one $n^{-1 / 2}\left(p_{n} \log n\right)^{1 / 2}$ that Ko et al. [13] obtained, where $p_{n} \leq n / 2$ and $p_{n} \rightarrow \infty$ as $n \rightarrow \infty$.

(2) Since LNQD sequences are strictly weaker than NA sequences, as mentioned in Section 1, Theorem 1 extends Theorem 2.1 in Jabbari et al. [7] from strictly stationary negatively associated setting to weighted LNQD case. In addition, by the analysis mentioned above, we know that the strong convergence rate $O(1) n^{-1 / 2} \log ^{1 / 2} n$ of $\sum_{i=1}^{n}\left(X_{i}-E X_{i}\right) / n$ is much faster than the relevant one $O(1) n^{-1 / 3}(\log n)^{2 / 3}$ Jabbari et al. [7] obtained only for the special case of geometrically decreasing covariances.

(3) For the sequence of extended negatively dependent (END, say) or wide orthant dependent (WOD, say) random variables, similar results can also be obtained.

Regarding the convergence rate $n^{-1 / 2} \log ^{1 / 2} n$ obtained in (5), we can give the following precise asymptotics.

Theorem 4. Let $\left\{X_{i}, i \geq 1\right\}$ be a sequence of identically distributed $L N Q D$ random variables with $E X_{1}=0$ and $E\left|X_{1}\right|^{2+\delta}<\infty$ for some $0<\delta \leq 1$, inf ${ }_{n \geq 1} \sigma_{n}^{2} / n>0$, $u(n) \leq C n^{-\theta}$ for some $\theta>1$, and

$$
\sigma^{2}:=E X_{1}^{2}+2 \sum_{i=2}^{\infty} \operatorname{Cov}\left(X_{1}, X_{i}\right)>0 .
$$

Then for $\beta>-1$, one has

$$
\lim _{\epsilon \searrow 0} \epsilon^{2 \beta+2} \sum_{n=2}^{\infty} \frac{(\log n)^{\beta}}{n} P\left(\left|S_{n}\right| \geq \epsilon \sigma \sqrt{n \log n}\right)=\frac{E|N|^{2 \beta+2}}{\beta+1},
$$

where $N$ denotes the standard normal random variable.

\section{Proofs}

Firstly, the following lemma is needed, which will be used in what follows.

Lemma 5 (see [10]). Let $\left\{X_{i}, i \geq 1\right\}$ be an LNQD sequence with zero mean and finite $u$ th moment. Then, for $u>2$, there exists a positive constant $C$ which only depends on $u$ such that

$$
E\left|\sum_{i=1}^{n} X_{i}\right|^{u} \leq C n^{u / 2} \max _{1 \leq i \leq n} E\left|X_{i}\right|^{u}
$$

for any $n \geq 1$

Next, we give some notations used later. Define $X_{n i}=X_{i}$ for $1 \leq i \leq n$ and $X_{n i}=0$ for $i>n$. Set $r_{n}=\left[n /\left(2 p_{n}\right)\right]+1$ and then define

$$
\begin{gathered}
Y_{n j}=\sum_{i=2(j-1) p_{n}+1}^{2(j-1) p_{n}+p_{n}} a_{n i}\left(X_{n i}-E X_{n i}\right), \\
Z_{n j}=\sum_{i=2(j-1) p_{n}+p_{n}+1}^{2 j p_{n}} a_{n i}\left(X_{n i}-E X_{n i}\right),
\end{gathered}
$$

for $j=1,2, \ldots, r_{n}$ and

$$
S_{1 n}=\sum_{j=1}^{r_{n}} Y_{n j}, \quad S_{2 n}=\sum_{j=1}^{r_{n}} Z_{n j} .
$$

Clearly, $\sum_{i=1}^{n} a_{n i}\left(X_{i}-E X_{i}\right)=S_{1 n}+S_{2 n}$ and $n<2 r_{n} p_{n} \leq 2 n$. Now, we can obtain the following lemma. 
Lemma 6. Let $\left\{X_{i}, i \geq 1\right\}$ be a sequence of stationary LNQD random variables with $\left|X_{i}\right| \leq M$ and let $\left\{a_{n i}: 1 \leq i \leq n, n \geq 1\right\}$ be a triangular array of numbers satisfying $\left|a_{n i}\right| \leq M_{1}$, where $M$ and $M_{1}$ are generic positive constants. If $0<\lambda p_{n} M M_{1} \leq$ $1 / 2$ for some $\lambda>0$, then on account of Definitions (9) and (10), one has

$$
\begin{aligned}
& E\left(\exp \left(\lambda S_{1 n}\right)\right) \leq \exp \left(C \lambda^{2} n\right), \\
& E\left(\exp \left(\lambda S_{2 n}\right)\right) \leq \exp \left(C \lambda^{2} n\right) .
\end{aligned}
$$

Proof. Without loss of generality, assume that $a_{n i} \geq 0$. Applying $E Y_{n j}=0$ and $\lambda p_{n} M M_{1} \leq 1 / 2$, we have by $\left|\lambda Y_{n j}\right| \leq$ $2 \lambda p_{n} M M_{1}$ that

$$
\begin{aligned}
E\left(\exp \left(\lambda Y_{n j}\right)\right) & =\sum_{k=0}^{\infty} \frac{E\left(\lambda Y_{n j}\right)^{k}}{k !}=1+\sum_{k=2}^{\infty} \frac{E\left(\lambda Y_{n j}\right)^{k}}{k !} \\
& \leq 1+E\left(\lambda Y_{n j}\right)^{2} \sum_{k=2}^{\infty} \frac{1}{k !} \\
& \leq 1+\lambda^{2} E Y_{n j}^{2} \leq \exp \left(\lambda^{2} E Y_{n j}^{2}\right) .
\end{aligned}
$$

Therefore, in terms of the result above and the concept of LNQD,

$$
\begin{aligned}
& \prod_{j=1}^{r_{n}} E\left(\exp \left(\lambda Y_{n j}\right)\right) \\
& \quad \leq \exp \left(\lambda^{2} \sum_{j=1}^{r_{n}} E Y_{n j}^{2}\right) \\
& \quad \leq \exp \left(M_{1}^{2} \lambda^{2} \sum_{j=1}^{r_{n}} \sum_{i=2(j-1) p_{n}+1}^{2(j-1) p_{n}+p_{n}} \operatorname{Var}\left(X_{n i}\right)\right) \\
& \quad \leq \exp \left(M_{1}^{2} \lambda^{2} \sum_{j=1}^{r_{n}} \sum_{i=2(j-1) p_{n}+1}^{2(j-1) p_{n}+p_{n}} E X_{1}^{2}\right) \\
& \quad \leq \exp \left(C \lambda^{2} n\right) .
\end{aligned}
$$

Also, since $a_{n i} \geq 0$ and $\lambda>0,\left\{\lambda Y_{n j}, 1 \leq j \leq r_{n}\right\}$ is LNQD. Therefore, from Lemma 3.1 in Ko et al. [13], we have

$$
E\left(\exp \left(\lambda S_{1 n}\right)\right) \leq \prod_{j=1}^{r_{n}} E\left(\exp \left(\lambda Y_{n j}\right)\right) .
$$

Combining (13) and (14), we can get the desired result. Similarly, we can get the same result for $S_{2 n}$. The proof is completed.

By Lemma 6, we can easily get the following result.

Lemma 7. Let $\left\{X_{i}, i \geq 1\right\}$ be a sequence of stationary LNQD random variables with $\left|X_{i}\right| \leq M$ and let $\left\{a_{n i}: 1 \leq i \leq n, n \geq 1\right\}$ be a triangular array of numbers satisfying $\left|a_{n i}\right| \leq M_{1}$, where
$M$ and $M_{1}$ are generic positive constants. If $0<\lambda p_{n} M M_{1} \leq$ $1 / 2$ for some $\lambda>0$, then, for any $0<\varepsilon<1$ and some $C_{1}>0$,

$$
\begin{aligned}
& P\left(\left|S_{1 n}\right|>\frac{n \varepsilon}{2}\right) \leq C_{1} \exp \left\{-\frac{n \varepsilon^{2}}{16 C}\right\}, \\
& P\left(\left|S_{2 n}\right|>\frac{n \varepsilon}{2}\right) \leq C_{1} \exp \left\{-\frac{n \varepsilon^{2}}{16 C}\right\} .
\end{aligned}
$$

Proof. Applying Markov inequality and Lemma 6, we obtain

$$
\begin{aligned}
& P\left(\left|S_{1 n}\right|>\frac{n \varepsilon}{2}\right) \\
& \quad=P\left(S_{1 n}>\frac{n \varepsilon}{2}\right)+P\left(-S_{1 n}>\frac{n \varepsilon}{2}\right) \\
& \quad \leq P\left(e^{\lambda S_{1 n}}>e^{\lambda n \varepsilon / 2}\right)+P\left(e^{-\lambda S_{1 n}}>e^{\lambda n \varepsilon / 2}\right) \\
& \quad \leq C_{1} \exp \left(C \lambda^{2} n-\frac{\lambda n \varepsilon}{2}\right) .
\end{aligned}
$$

Optimizing the exponent in the term of this upper-bound, we find that $\lambda=\varepsilon /(4 C)$, so that this exponent becomes equal to $-n \varepsilon^{2} /(16 C)$ as desired. The proof is completed.

Lemma 8 (see [10]). Under the conditions of Theorem 4, one has

$$
\frac{\sigma_{n}^{2}}{n} \longrightarrow \sigma^{2}, \quad \frac{S_{n}}{\sigma_{n}} \longrightarrow N \quad \text { in distribution, }
$$

where $N$ is a standard normal random variable.

Lemma 9 (see [12]). Under the conditions of Theorem 4, we have for $x \in R$

$$
\sup _{x}\left|P\left(\frac{S_{n}}{\sigma_{n}}<x\right)-\Phi(x)\right| \leq C n^{-\delta /(2+3 \delta)},
$$

where $\Phi(x)$ denotes the standard normal distribution function.

Based on the above lemmas, the proofs of Theorems 1 and 4 can be given as follows.

Proof of Theorem 1. Let $\lambda>0$ which satisfies $\lambda p_{n} M M_{1} \leq 1 / 2$; then it follows from Lemma 7 that

$$
\begin{aligned}
P\left(\left|S_{n}\right|>n \varepsilon\right) & \leq P\left(\left|S_{1 n}\right|>\frac{n \varepsilon}{2}\right)+P\left(\left|S_{2 n}\right|>\frac{n \varepsilon}{2}\right) \\
& \leq C_{1} \exp \left\{-\frac{n \varepsilon^{2}}{16 C}\right\},
\end{aligned}
$$

which completes the proof. 
Proof of Theorem 4. Without loss of generality, set $\sigma=1$ in what follows. Since

$$
\begin{gathered}
\epsilon^{2 \beta+2} \sum_{n=2}^{\infty} \frac{(\log n)^{\beta}}{n} P\left(\left|S_{n}\right| \geq \epsilon \sqrt{n \log n}\right) \\
=\epsilon^{2 \beta+2} \sum_{n=2}^{\infty} \frac{(\log n)^{\beta}}{n}\left\{P\left(\left|S_{n}\right| \geq \epsilon \sqrt{n \log n}\right)\right. \\
-P(|N| \geq \epsilon \sqrt{\log n})\} \\
+\epsilon^{2 \beta+2} \sum_{n=2}^{\infty} \frac{(\log n)^{\beta}}{n} P(|N| \geq \epsilon \sqrt{\log n}),
\end{gathered}
$$

it is sufficient to prove that

$$
\begin{gathered}
\lim _{\epsilon \searrow 0} \epsilon^{2(\beta+1)} \sum_{n=2}^{\infty} \frac{(\log n)^{\beta}}{n} P(|N| \geq \epsilon \sqrt{\log n})=\frac{E|N|^{2(\beta+1)}}{\beta+1}, \\
\lim _{\epsilon \searrow 0} \epsilon^{2(\beta+1)} \sum_{n=2}^{\infty} \frac{(\log n)^{\beta}}{n} \mid P\left(\left|S_{n}\right| \geq \epsilon \sqrt{n \log n}\right) \\
-P(|N| \geq \epsilon \sqrt{\log n}) \mid=0
\end{gathered}
$$

for any $\beta>-1$.

To prove (21), we need only to show that

$$
\lim _{\epsilon \searrow 0} \epsilon^{2(\beta+1)} \sum_{n=2}^{\infty} \frac{(\log n)^{\beta}}{n} P(N \geq \epsilon \sqrt{\log n})=\frac{E|N|^{2(\beta+1)}}{2(\beta+1)},
$$

by noting $P(|N| \geq x)=2 P(N \geq x)$ for any $x>0$. It is easy to observe that

$$
\begin{aligned}
& \lim _{\epsilon \searrow 0} \epsilon^{2(\beta+1)} \sum_{n=2}^{\infty} \frac{(\log n)^{\beta}}{n} P(N \geq \epsilon \sqrt{\log n}) \\
& =\lim _{\epsilon \searrow 0} \epsilon^{2(\beta+1)} \int_{2}^{\infty} \frac{(\log x)^{\beta}}{x} P(N \geq \epsilon \sqrt{\log x}) d x \\
& =\lim _{\epsilon \searrow 0} \int_{\epsilon}^{\infty} 2 y^{2 \beta+1} P(N \geq y) d y \\
& =\frac{E|N|^{2(\beta+1)}}{2(\beta+1)}
\end{aligned}
$$

for any $\beta>-1$, which implies (21).
Next, we will prove (22). Let $J(\epsilon)=\exp \left(M / \epsilon^{2}\right)$, where $M>4$ and $0<\epsilon<1 / 4$. Obviously,

$$
\begin{aligned}
& \sum_{n=2}^{\infty} \frac{(\log n)^{\beta}}{n} \mid P\left(\left|S_{n}\right| \geq \epsilon \sqrt{n \log n}\right) \\
& \quad-P(|N| \geq \epsilon \sqrt{\log n}) \mid \\
& =\sum_{n \leq J(\epsilon)} \frac{(\log n)^{\beta}}{n} \mid P\left(\left|S_{n}\right| \geq \epsilon \sqrt{n \log n}\right) \\
& +\sum_{n>J(\epsilon)} \frac{(\log n)^{\beta}}{n} \mid P\left(\left|S_{n}\right| \geq \epsilon \sqrt{n \log n}\right) \\
& \left.\quad+=I_{1}+I_{2}+I_{3} ;|N| \geq \epsilon \sqrt{\log n}\right) \mid \\
& \leq \sum_{n \leq J(\epsilon)} \frac{(\log n)^{\beta}}{n} \mid P\left(\left|S_{n}\right| \geq \epsilon \sqrt{n \log n}\right) \\
& +\sum_{n>J(\epsilon)} \frac{(\log n)^{\beta}}{n} P\left(\left|S_{n}\right| \geq \epsilon \sqrt{n \log n}\right) \\
& -P(|N| \geq \epsilon \sqrt{\log n}) \mid
\end{aligned}
$$

thus it suffices to prove that

$$
\begin{gathered}
\lim _{\epsilon \searrow 0} \epsilon^{2(\beta+1)} I_{1}=0, \quad \lim _{\epsilon \searrow 0} \epsilon^{2(\beta+1)} I_{2}=0, \\
\lim _{\epsilon \searrow 0} \epsilon^{2(\beta+1)} I_{3}=0,
\end{gathered}
$$

respectively. We consider firstly $I_{1}$. Set $\Delta_{n}=\sup _{x} \mid P\left(\left|S_{n}\right| \geq\right.$ $x \sqrt{n})-P(|N| \geq x) \mid$. Noticing Lemma 8 , we have $\Delta_{n} \rightarrow 0$ as $n \rightarrow \infty$. It follows that

$$
\begin{aligned}
& \lim _{\epsilon \searrow 0} \epsilon^{2(\beta+1)} I_{1} \\
& \quad \leq \lim _{\epsilon \searrow 0} \epsilon^{2(\beta+1)} \sum_{n \leq J(\epsilon)} \frac{(\log n)^{\beta}}{n} \Delta_{n} \\
& \quad=\lim _{\epsilon \searrow 0} \epsilon^{2(\beta+1)}(\log J(\epsilon))^{\beta+1} \frac{1}{(\log J(\epsilon))^{\beta+1}} \sum_{n \leq J(\epsilon)} \frac{(\log n)^{\beta}}{n} \Delta_{n} \\
& \quad \leq \lim _{\epsilon \searrow 0} M^{\beta+1} \frac{1}{(\log J(\epsilon))^{\beta+1}} \sum_{n \leq J(\epsilon)} \frac{(\log n)^{\beta}}{n} \Delta_{n} \longrightarrow 0,
\end{aligned}
$$


which implies that $\lim _{\epsilon \downarrow 0} \epsilon^{2(\beta+1)} I_{1}=0$. Turn to $I_{2}$. By Lemma 9, it follows that

$$
\begin{aligned}
P\left(\left|S_{n}\right| \geq \epsilon \sqrt{n \log n}\right) & \\
\leq & \left|P\left(\left|\frac{S_{n}}{\sigma_{n}}\right| \geq \frac{\epsilon \sqrt{n \log n}}{\sigma_{n}}\right)-P\left(|N| \geq \frac{\epsilon \sqrt{n \log n}}{\sigma_{n}}\right)\right| \\
& +P\left(|N| \geq \frac{\epsilon \sqrt{n \log n}}{\sigma_{n}}\right) \\
\leq & C n^{-\delta /(2+3 \delta)}+2 P\left(N \geq \frac{\epsilon \sqrt{n \log n}}{\sigma_{n}}\right) \\
\leq & C n^{-\delta /(2+3 \delta)}+C n^{-\epsilon^{2} / 2} \leq C n^{-\epsilon^{2} / 2},
\end{aligned}
$$

for sufficiently small $\epsilon$. Thus we obtain

$$
\begin{aligned}
& \lim _{M \rightarrow \infty} \epsilon^{2(\beta+1)} I_{2} \\
& =\lim _{M \rightarrow \infty} \epsilon^{2(\beta+1)} \sum_{n>J(\epsilon)} \frac{(\log n)^{\beta}}{n} P\left(\left|S_{n}\right| \geq \epsilon \sqrt{n \log n}\right) \\
& \leq C \lim _{M \rightarrow \infty} \epsilon^{2(\beta+1)} \sum_{n>J(\epsilon)} \frac{(\log n)^{\beta}}{n} \exp \left(-\frac{\epsilon^{2}}{2} \log n\right) \\
& \leq C \lim _{M \rightarrow \infty} \epsilon^{2(\beta+1)} \int_{J(\epsilon)}^{\infty} \frac{(\log x)^{\beta}}{x} \exp \left(-\frac{\epsilon^{2}}{2} \log x\right) d x \\
& \leq C \lim _{M \rightarrow \infty} \epsilon^{2(\beta+1)} \int_{M / 2 \epsilon^{2}}^{\infty} y^{\beta} \exp \left(-\frac{\epsilon^{2}}{2} y\right) d y \\
& \leq C \lim _{M \rightarrow \infty} \int_{M / 4}^{\infty} t^{\beta} e^{-t} d t \longrightarrow 0 .
\end{aligned}
$$

Hence, $\lim _{\epsilon \succ 0} \epsilon^{2(\beta+1)} I_{2} \rightarrow 0$ when $M \rightarrow \infty$. On the other hand, noting that $M>4$ and $0<\epsilon<1 / 4$ imply $J(\epsilon)-1 \geq$ $\sqrt{J(\epsilon)}$, we can obtain

$$
\begin{aligned}
& \lim _{\epsilon \searrow 0} \epsilon^{2(\beta+1)} I_{3} \\
& \quad \leq \lim _{\epsilon \searrow 0} \epsilon^{2(\beta+1)} \int_{J(\epsilon)}^{\infty} \frac{(\log x)^{\beta}}{x} P(|N| \geq \epsilon \sqrt{\log x}) d x
\end{aligned}
$$

$$
\begin{aligned}
& \leq \lim _{\epsilon \searrow 0} \epsilon^{2(\beta+1)} \int_{J(\epsilon)-1}^{\infty} \frac{(\log x)^{\beta}}{x} P(|N| \geq \epsilon \sqrt{\log x}) d x \\
& \leq \lim _{\epsilon \searrow 0} \epsilon^{2(\beta+1)} \int_{\sqrt{J(\epsilon)}}^{\infty} \frac{(\log x)^{\beta}}{x} P(|N| \geq \epsilon \sqrt{\log x}) d x \\
& \leq C \lim _{\epsilon \searrow 0} \int_{\epsilon \sqrt{M /\left(2 \epsilon^{2}\right)}}^{\infty} y^{2 \beta+1} P(|N| \geq y) d y \\
& \leq C \int_{\sqrt{M / 2}}^{\infty} y^{2 \beta+1} P(|N| \geq y) d y \longrightarrow 0 \text { as } M \longrightarrow \infty,
\end{aligned}
$$

uniformly for $0<\epsilon<1 / 4$. Thus we have $\lim _{\epsilon \succ 0} \epsilon^{2(\beta+1)} I_{3} \rightarrow$ 0 , when $M \rightarrow \infty$. Combining the earlier results together yields (26). The proof is complete.

\section{Conflict of Interests}

The authors declare that there is no conflict of interests regarding the publication of this paper.

\section{Acknowledgments}

This research is supported by the Scientific Research Foundation of Guangxi Colleges and Universities (no. 2013LX019), the National Science Foundation of China (11061007), and the Natural Science Foundation of Guangxi (2011GXNSFA018133).

\section{References}

[1] E. L. Lehmann, "Some concepts of dependence," Annals of Mathematical Statistics, vol. 37, no. 5, pp. 1137-1153, 1966.

[2] C. M. Newman, "Asymptotic independence and limit theorems for positively and negatively dependent random variables," in Statistics and Probability, Y. L. Tong, Ed., vol. 5, pp. 127-140, Institute of Mathematical Statistics, Hayward, Calif, USA, 1984.

[3] K. Joag-Dev and F. Proschan, "Negative association of random variables with applications," Annals of Statistics, vol. 11, no. 1, pp. 286-295, 1983.

[4] S. Yang and Y. Wang, "Strong consistency of regression function estimator for negatively associated samples," Acta Mathematicae Applicatae Sinica, vol. 22, no. 4, pp. 522-530, 1999 (Chinese).

[5] T.-S. Kim and H.-C. Kim, "On the exponential inequality for negative dependent sequence," Communications of the Korean Mathematical Society, vol. 22, no. 2, pp. 315-321, 2007.

[6] S. H. Sung, "A note on the exponential inequality for associated random variables," Statistics and Probability Letters, vol. 77, no. 18, pp. 1730-1736, 2007.

[7] H. Jabbari, M. Jabbari, and H. A. Azarnoosh, "An exponential inequality for negatively associated random variables," Electronic Journal of Statistics, vol. 3, pp. 165-175, 2009.

[8] G. Xing, S. Yang, A. Liu, and X. Wang, "A remark on the exponential inequality for negatively associated random variables," Journal of the Korean Statistical Society, vol. 38, no. 1, pp. 53-57, 2009.

[9] S. H. Sung, "An exponential inequality for negatively associated random variables," Journal of Inequalities and Applications, vol. 2009, Article ID 649427, 7 pages, 2009. 
[10] L.-X. Zhang, "A functional central limit theorem for asymptotically negatively dependent random fields," Acta Mathematica Hungarica, vol. 86, no. 3, pp. 237-259, 2000.

[11] H. Kim and T. Kim, "A central limit theorem for general weighted sum of LNQD random variables and its application," Communications of the Korean Mathematical Society, vol. 20, no. 3, pp. 531-538, 2005.

[12] J. Wang and L. Zhang, "A Berry-Esseen theorem for weakly negatively dependent random variables and its applications," Acta Mathematica Hungarica, vol. 110, no. 4, pp. 293-308, 2006.

[13] M.-H. Ko, Y.-K. Choi, and Y.-S. Choi, "Exponential probability inequality for linearly negative quadrant dependent random variables," Communications of the Korean Mathematical Society, vol. 22, no. 1, pp. 137-143, 2007. 


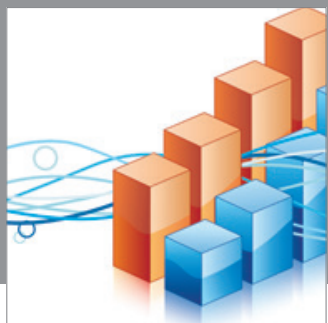

Advances in

Operations Research

mansans

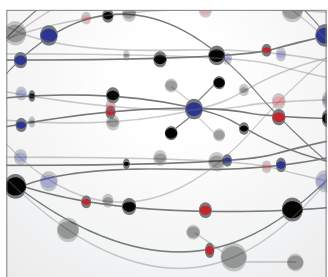

The Scientific World Journal
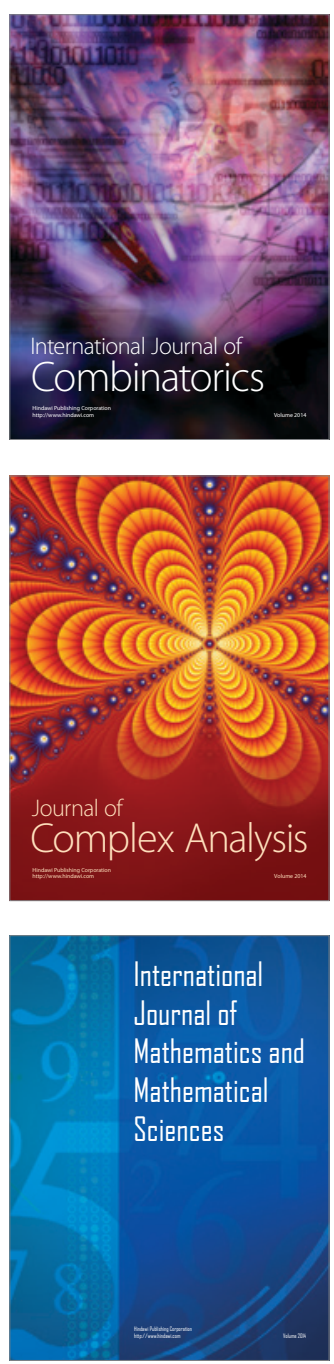
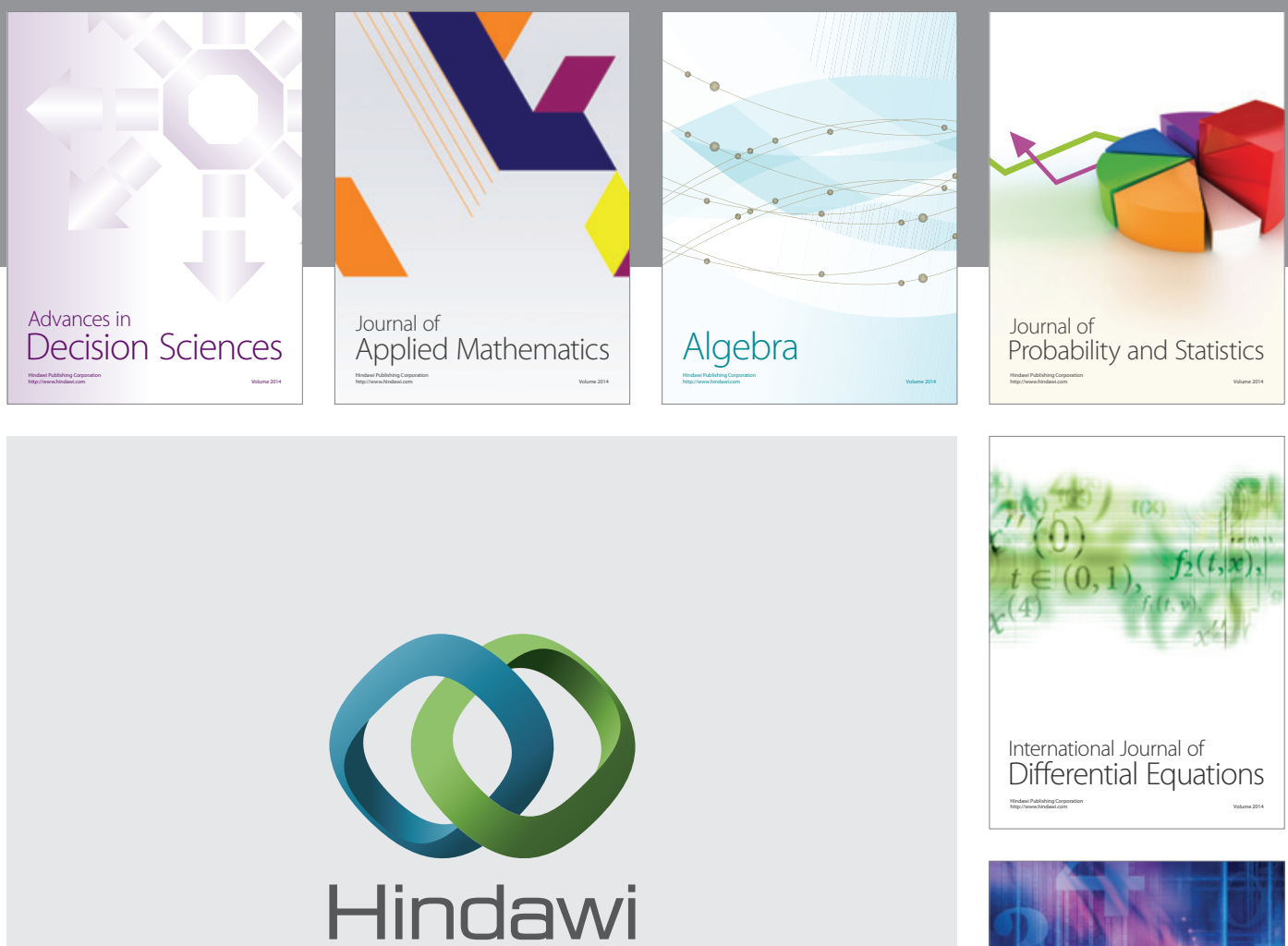

Submit your manuscripts at http://www.hindawi.com
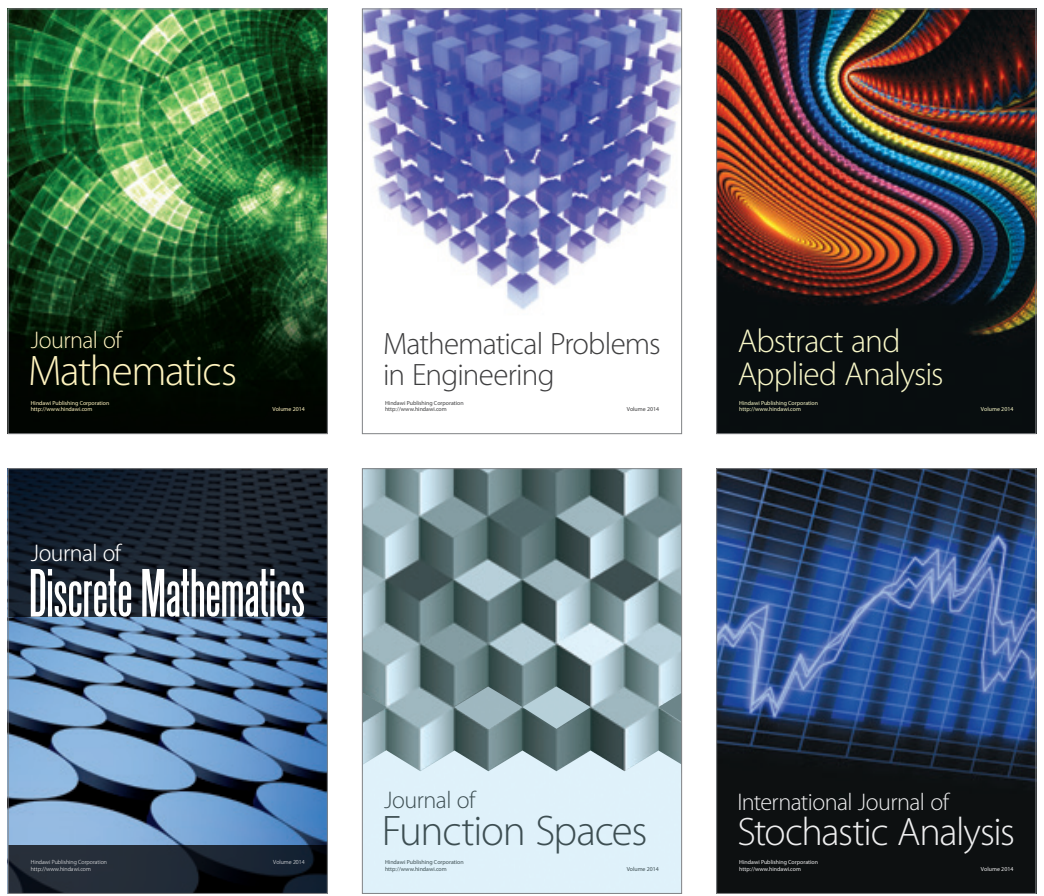

Journal of

Function Spaces

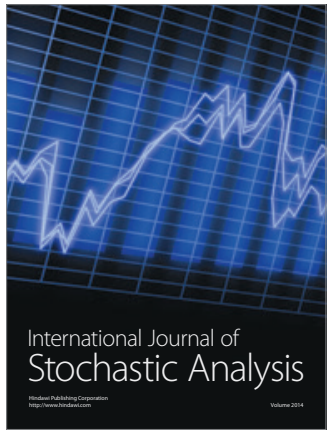

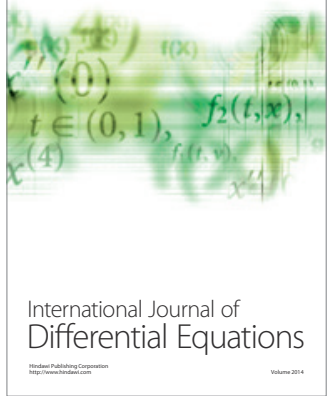
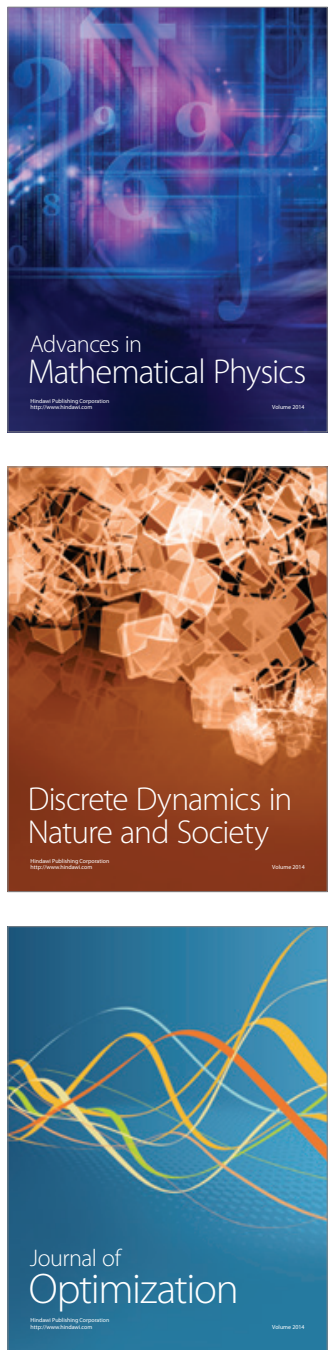